\title{
Value Theory and Online Video Gaming
}

\author{
Derek A. Burrill \\ Department of Media and Cultural Studies, University of California, Riverside, CA, USA \\ Email: derekb@ucr.edu
}

Received 1 June 2014; revised 2 July 2014; accepted 27 July 2014

Copyright (C) 2014 by author and Scientific Research Publishing Inc.

This work is licensed under the Creative Commons Attribution International License (CC BY). http://creativecommons.org/licenses/by/4.0/

(c) (;) Open Access

\begin{abstract}
Online video gaming has been an important object of study because of its complex social and cultural processes. However, few studies exist in the discipline of games studies that inspect actual gamers' concepts of online gaming value. Value theory exists in other disciplines (economics, sociology, axiology, etc.), but has not yet been fully explored in relation to online gaming worlds. This study seeks to inspect how gamers define value and how this is expressed through their online gaming experiences. Additionally, the survey seeks to establish a link between the internal value of the games and the concept of value in the external, real world. The analysis of the survey in this paper shows that gamers establish value in online gaming in relation to value in the external.
\end{abstract}

\section{Keywords}

Value Theory, Online Gaming, Exchange, Perceived Time

\section{Introduction}

In the past few years, several attempts have been made to account for value in online videogaming. Whether value is judged according to economic, social or psychological systems, these studies have theorized how online gaming functions as a discrete space in which something (an experience, the quality of time spent, the economic structures at work in the online world, etc.) is intrinsically and extrinsically valued. Often, the objects of study are figurative (skills, levels of accomplishment, etc.) or concrete (weapons, property, etc.) factors that are either exchanged or produced in the world of game, with a corresponding value based on the expense and difficulty of production in the real world (see Malaby, 2006). These studies are important because they establish that systems of exchange, pleasure, and desire operate similarly to systems in the real and are usually based on a reciprocal value exchange rate. Games researchers, and those interested in online worlds in general, should be familiar with the media attention in recent years on sweatshops where workers punch keys for hours on end in order to complete mundane tasks so that gamers can purchase these products and skill sets for use in the games without having to actually complete the tasks themselves. As far as advanced capitalism goes, this is hardly surprising.

This study seeks to theorize how the term value is actually used in relation to gamers' sense of their online 
time and experiences. To this end, this study borrows from economic models of value, but seeks more so to uncover the quality of time spent gaming online and how this is seen as valuable. The sections of this piece are as follows: an overview of the term value and its relationship to the larger area of study called value theory, a summary of an online survey featuring questions regarding value, an analysis of the findings of the survey and specific reactions from the respondents, and a conclusion.

\subsection{Value Theory}

Value, as a concept both concrete and figurative, has relevance to a number of areas including, but not limited to, economics, law, ethics and axiology, sociology, psychology, color theory, and art. However, in each of these disciplines, value has two fundamental qualities. First, value is based on exchange. Second, value oscillates between subjective and predicate understandings. These two qualities are mutually constative- they produce and maintain each other as an imbricated system. In classical economics, for instance, value originates in production, so that however much time and effort a worker puts into producing something-a wooden chair, for example — dictates that thing's (e.g., the chair's) value on the market (Smith, 1937). This approach was introduced in Adam Smith's The Wealth of Nations, where the author argues that labor is the defining factor in value determination. Additionally, Marx (1968), in The Communist Manifesto, found that value is manifested in how much labor is needed to produce said chair. Of course, Marx sought to uncover the fundamental problems within the conditions of labor and how value then becomes a product of inequity and oppression. In this way, the unit of analysis for classical economics is social class, thus value is a direct product of the configuration of power within capitalism. On the other hand, in neoclassical economics, value is not based on production, but on exchange-literally how, when, and where the products are bought, sold or traded and the market forces that control this exchange (Creedy, 2007). In this sense, neoclassical economics uses the individual as the central unit of analysis, marking a distinct shift toward a more subjective notion of value. A person could, for example, find a wooden chair at an antique store that costs significantly more than it cost to produce, but the chair has accrued a great deal of value because of its workmanship, age, appearance, etc. Yet, this chair, while to those who value these qualities, would be worth the cost, could be viewed as inferior to those who do not share the owner/ buyers' predicate value system. Thus, value and exchange operate on many levels, in micro-worlds and macro-worlds. This is particularly obvious in online gaming worlds, where small groups often dictate localized value systems even though macro-economic systems may differ.

An offshoot of value theory in economics is known as the Energy Theory of Value, where the laws of thermodynamics are applied to value systems (Odum, 1983). According to this theory, values in systems are dictated by the three laws of thermodynamics: energy is conserved, entropy cannot decrease in isolated systems, and entropy equals zero when absolute temperature is zero. These laws describe how energy operates in relationship to factors such as temperature, force, friction, etc. In Energy Theory, the basic physical laws form the unit of analysis, so value and the mode of exchange are dictated not by social classes or individuals (although they are of course involved in exchange), but instead by flows of energy within systems. This is largely due to what Odum (1983), a pioneer in the field, calls the maximum power principle, or, how systems follow patterns of behavior based on the transformation of energy into power efficiently and quickly so that organisms can remain competitive. While Energy Theory is largely the product of non-economists, it has been useful as a means of understanding how value operates systemically and how value is a product of external and internal forces that generally seek to create stability and equilibrium.

Finally, value, in less economic terms, simply means the relative or fixed importance of something. This has bearing on sociology, psychology, ethics, and the law in that a society or culture shares certain ethical and moral codes so that the health of that group can be maintained and so that internal disruptions can be solved in an agreed upon fashion. In this sense, values can be said to be mutative and can shift at cultural levels, yet are often quite solid for the individual. Large-scale events (the recent global recession, for example) can cause wide-scale shifts that can alter the values of a nation without disrupting the day-to-day values of its citizenry. Thus, value has been theorized as based on production, exchange, and energy flow, as well as by cooperation, namely between the individual and the larger culture.

Value as a quality in gaming was originally theorized by both Caillois and Huizinga. Huizinga (1945), in Homo Ludens, finds four identifiable structures inherent to all forms of play: 1) play is for itself, it has no external goal, 2) play exists outside the scope of ordinary life, 3) play operates within fixed boundaries of space and time, with its own set of rules, and 4) play is labile. Additionally, Caillois (1961), in Man, Play and Games finds 
that "play and life are constantly and universally antagonistic to each other” (p. 4). While Huizinga and Caillois differ in their approach to the study of play and games, both clearly devalue games in the face of more important pursuits, such as labor. While both find that the playspace is a culturally significant arena with its own rules and logic, both write from a clearly Modernist position, so that the divide between high and low culture must be necessarily maintained. The view that play and games clearly possess cultural value found a historical home in Postmodernism, and in the academy in the areas of sports studies (Messner, 1992), performance studies (Phelan \& Lane, 1998), and queer and gender studies (Black, 2001) ${ }^{1}$. With the birth of digital games studies, a de facto sense that games and play have value was established by the quick growth of the field and the rise of research centers and departments devoted to the field. Two recent studies, both published in the same issue of Games and Culture, deal with value, although they do not address actual player experience. Malaby (2006), in Parlaying Value: In and Beyond Virtual Worlds, theorizes the structure of capital in virtual worlds, showing that a third category of value (other than market value for commodities and currencies and the social value of networks in and out of the virtual), cultural capital, if studied properly, will reveal how all three forms of capital operate in virtual worlds. While his analysis is excellent, it is not distinctly concerned with what actual players consider valuable - in concrete and abstract terms-and how this value relates to value orientation outside the virtual. Castronova's (2006) On the Research Value of Large Games identifies MMORPG's as valuable, complex, and noteworthy objects of sociological and theoretical social/cultural research. Again, this is a trenchant analysis, but does not seek to establish individual players' notions of value.

\subsection{Objective}

While past research has been informative in exploring notions of value, these studies lack actual players' insights into value in online gaming. The research conducted for this paper explores gamers' notions of value in relation to online gaming in order to establish whether players do indeed value their time and experiences online and how they express this value.

\subsection{Research Questions}

Three qualitative research questions structured the study reported below.

1) How much do online gamers value their gaming?

2) Do online gamers value gaming more than, less than, or the same as time spent working, traveling, or in leisure pursuits?

3) To what degree is the amount of time spent gaming factored into gamers' valuation of their online gaming?

\section{Study}

\subsection{Participants}

The participants in this study were 112 adults; $68 \%$ of respondents self-identified as male, $23 \%$ self-identified as female, and the remainder identified themselves as "other". Participants were contacted through three online gaming discussion groups.

\subsection{Method}

\section{Materials}

The survey focused on online gaming instead of console or portable gaming because of the more social nature of online games, as well as to avoid involving the general assumption that console/portable gaming is seen as a less meaningful pursuit amongst gamers and the non-gaming public (Newman, 2002). Participants answered four open-ended questions:

1) How do you define "value"?

2) How much value do you find in playing online games?

3) How do you rate this value in comparison to other pursuits such as work, leisure, travel, etc.?

4) Is time a factor in your rating of game play value? How so?

${ }^{1}$ However, one could point to the work of Mikail Bakhtin on carnival in the first half of the twentieth century as a significant treatment of related topics such as festivity, the carnivalesque and play. 
Question 1 allowed the respondent to define value individually and was used to gauge responses to the next three questions. In Question 2, participants provided a largely qualitative assessment of value; and in Question 3, participants provided a primarily quantitative assessment of value. Question 4 was meant as a control for the level and depth of the respondents' play.

\subsection{Procedure}

The study employed a survey methodology and a purposive sampling method to target online gamers as participants. The survey was given over a period of two weeks during July of 2010. It was posted to three online gaming discussion groups, was voluntary, in English, anonymous and did not offer incentives or rewards. The only demographic information participants provided was gender. Participants were informed that their responses would be used for academic purposes.

\subsection{Results}

The 112 responses to each question varied in length and complexity. As an overview of the responses, participants generally answered Question 1 in terms not related to gaming; instead answers were largely either economically- or ethically-based. Question 2 was answered largely as a means to prove the worthiness of gaming pursuits. Question 3 was answered usually in direct correspondence to the terms presented in the question's wording (work, leisure, travel) and occasionally featured other pursuits (sports, socializing in the real world, and other personal interests). Question 4 was the least varied with nearly all respondents finding a direct correspondence between the amount of time played and the quality and amount of pleasure received (more time equaled more pleasure).

As noted in the introduction, value can be theorized in four ways: production, exchange, energy flow, and cooperation. Participants' responses were examined for references to these aspects of value within the context of online gaming.

Question 1: How do you find value?

Question 1 featured answers that traversed each of these conceptions, with nearly $40 \%$ focusing on production and exchange, although usually not in those distinct words. One respondent wrote:

Value is based on how much something is worth. It's [sic] monetary value. What someone would pay for it.

And another wrote:

How much something costs.

And another wrote:

The way something is priced.

The remainder of this $40 \%$ used similar terminology; "cost”, "monetary value”, and "worth", with "price" being the most common. The remaining $60 \%$ defined value in more sociological or ethical terms, often using familiar objects as a means of example. One respondent wrote:

I define value by what is valuable to me, not what other people think of as valuable. Sometimes I just don't get why some people think certain things are valuable, although sometimes I understand because I collect comix [sic] and some people don't get that.

Another respondent wrote:

A thing that your parents teach you.

Another wrote:

What you believe in.

Question 2: How much value do you find in playing online games?

This question generated a variety of responses. Nearly $50 \%$ of respondents sought to somehow validate the value of gaming, with an emphasis on an adversarial relationship to those that consider gaming "trivial" or "a waste of time”. One respondent wrote:

Somehow I knew this question would come up when I decided to take this survey. It's what pisses me off about how "experts" treat us. I don't think it has anything to do with how much "value" games have it's about how you spend the time. Sometimes I just want to have fun, sometimes I want to have a tough experience, sometimes I want to impress my friends and frag hard.

Another wrote:

My time gaming is VERY valuable to me. It's a way for me to get away from my daily crap. Unfortunately, 
most people around me, other then [sic] my gaming friends don’t get it. So I try to talk to them and show them and they usually don't care.

Another wrote:

I wish my parents would see what I can do when I rock. Then maybe they would buy me more games. :)

The remaining $50 \%$ of the respondents had a great deal to say about the quality of play in relation to value; however the responses reflected the individualized definitions of value and thus were not able to be meaningfully grouped. For instance, one respondent wrote:

The value of my online gaming depends on who I am playing with. Sometimes when the other players are new or I don't know them, it's not as fun. Or there is always someone there who is trying to be a pain. Then I just quit and go back later. But if I am with my mates then we have goodtimes.

Another wrote:

My day job sucks and my friends suck, so gaming has a really high value for me. It’s just a better time.

Another wrote:

I sometime [sic] think I was born 500 years ago because Everquest makes more sense to me then everyday life. Its [sic] more romantic and interesting. So, I find more value from gaming then from life.

Question 3: How do you rate this value in comparison to other pursuits such as work, leisure, travel, etc.?

This question was asked in order to establish how gaming ranks in comparison to other activities. Surprisingly, the majority of respondents found gaming to not only have more value than other pursuits, but to be of significantly more value. Ninety-three percent (93\%) found gaming to be of more value, and the remainder found gaming to have as much value as other pursuits. Work overwhelmingly ranked as least valuable (except in the case of one respondent, who incidentally works for a gaming company), with leisure, travel, and other pursuits named specifically by respondents still ranking below gaming. Certain interesting exceptions did occur, such as when respondents found inherent similarities between playing sports and gaming. One respondent wrote:

Gaming is almost as good as playing soccer. I like them both at different times for different reasons. They are both the most valuable things for me.

But, generally, most respondents favored gaming over other pursuits. For example:

Work sucks. I don't travel hardly ever and I spend all of my leisure time playing games. So gaming is the most valuable thing to me.

Another wrote:

The thing I value about gaming is that it lets me forget all about work and my wife and my kids. But, its [sic] not like I don't like work or my wife or my kids, but sometimes I need a break from them and there is nothing better then hanging out with friends and blowing stuff up. And I can meet people I never would.

Another wrote:

I like work and I like to travel, but let's face it, when I'm in-game its like fun work and travel at the same time.

Question 4: Is time a factor in your rating of game play value? How so?

Participants answered this question in one of two ways. 56\% answered in terms of how much time certain tasks took in certain games (many specific games were named in this series of responses) and how the relation between time and task difficulty altered value. On the other hand, $44 \%$ answered, in terms of the quality of time spent playing and how that related to perceived time (how much time would elapse before the player was aware many hours had passed or became bored or tired). From the first group, one respondent wrote:

I think a game is valuable if you can get a lot done in [a] short amount of time. I don't like too many repetitive tasks. Also, I feel like my time is better spent if more people are actually playing. I don't like it when it's a deadzone [sic].

Another wrote:

I quit playing Morrowind because everything was way too spread out and there wasn't enough to do. And the battle sequences were really hard to navigate.

Another wrote:

If I could get as much done in my real life as I can when I'm playing, I would be a very happy guy. So, I value playing if it's time well-spent [sic] and I feel like I accomplished something.

The second group of respondents found value if time somehow "disappeared". All in this group valued games that took long periods of time and filled up many hours without seeming to do so. One respondent wrote:

I dig it when I can play for like 10 or 12 hours and it seemed like no time at all passed. Its [sic] like the exact 
opposite of standing in line at a bank. And I don't mind spending that much time at all.

Another wrote:

Value = how much I DON'T [respondent's emphasis] realize how much time I just wasted. $($ )

Another wrote:

If I have enuff [sic] time, then I want to be able to totally disappear for a long time. To make sure I can just be there. That's why Everquest is so f-ing [sic] sweet.

\section{Discussion}

The purpose in conducting this survey was to inspect not only what gamers consider valuable but also how they conceive of value as an expression of their gaming experiences and time. Little research has been done in this area from the standpoint of gaming studies. A discussion of various forms of value theory is necessary in order to establish how the term has been theorized and how the term shifts over various disciplines. Although this survey is limited in scope, the patterns that emerged from the responses are useful as models for further study, as well as for the theorization of more general tendencies of gamers. Additionally, the results suggest that gamers actively discuss value and have shared understandings of what constitutes gaming value in a variety of dimensions, not just in a monetary sense.

In Question 1, the $40 \% / 60 \%$ split indicates that value is largely seen as either economic or ethical, even when gamers were aware that the survey was about gaming. It also means that value can be a highly personal concept, as evidenced by the high number of respondents who located value as a subjective quality. This speaks to the personal and communal nature of online games in general, and more specifically to the structural features of computers - they are meant to be used by one person, but are seen also as portals to substantive social worlds. Thus, value for these gamers is defined by forces that define the games themselves, suggesting that value functions similarly in games as it does in the real, although gaming and its fantasy components often seem to suggest the opposite, particularly when it comes to violent content. The substantial portion of respondents who described value in terms of economics (production and exchange) also suggests a personal acknowledgement of the market-driven nature of the games, online labor practices, and a general sense of games and gaming within capitalism. In short, value as defined by the respondents tended to fall within two fairly constrained categories, economic and ethical, although verbiage and terminology varied. Only two responses included mentions of gaming in their definition of value.

The responses to Question 2 suggest a "chip-on-the-shoulder" mentality remains as an important factor in establishing value in online gaming. Slightly more than half of the respondents stated that a great deal of value was found in gaming, but that this was underappreciated and misunderstood by non-participants. This indicates that regardless of the enormous amounts of money, time and experience exchanged in the spaces and networks of the games, two situations appear to continue to be played out: first, that the gamers themselves suffer from some type of guilt associated with either the amount of time spent gaming, or the validity of said pursuits in relation to other activities in the real world, and second, that gamers identify themselves as inherently separate from non-gamers, in possession of privileged information, skills, and experiences. The guilt complex also clearly indicates that non-gamers still have a negatively-biased opinion of gaming and that, as an emergent cultural form, it is still distrusted and misunderstood. Therefore, more than half of the respondents articulated their own guilt, but also enunciated, through this guilt and frustration, the surrounding culture's biases and how these biases operate as a force that differentiates and sequesters.

The remainder of the respondents to Question 2 found a great deal of value in playing online games, often expressed at a deeply-felt, personal level. Many expressed that online gaming had a value that exceeded that of their everyday lives, signaling not only the expressive power and complexity of online worlds, but also that these worlds may in fact attract people who are fundamentally unhappy with their everyday lives and are seeking to replace the everyday with the fantastic. Or, on the other hand, online gaming can potentially attract anyone (with access) at certain times when the subject is dissatisfied with the everyday. This is hardly surprising, as media and communications studies has historically found that media serve as a powerful cathartic and substitutive means of supplanting everyday stress, anxiety, and displeasure (Ball-Rokeach \& DeFleur, 1976; Katz \& Foulkes, 1962). Regardless, the vast majority of respondents found high levels of value in online gaming.

The respondents to Question 3 overwhelmingly found that online gaming had more value than other pursuits such as work, travel, leisure, etc. Of course, this survey was conducted with the notion that all respondents were 
familiar with online gaming, if not very involved. However, the level of frustration with other pursuits (or the lack of interest in them) was surprising. Many felt anger toward their work, frustration with family and nongamers, and a strong affinity for gaming as a means of expressing this frustration and anger. Again, this speaks to the strong cathartic effect of gaming. Many players expressed pleasure in being able to express themselves through violent and hyper-active encounters with other gamers. Others expressed a strong fraternal bond with their playing buddies, so much so that real world relationships seemed to often be at odds with their gaming time and relations. All of this points to a central issue; respondents found that gaming had not only a higher value than other pursuits, but its value was judged on a scale of opposition to other pursuits, so that online gaming's inherent value was not necessarily expressed as much as its relational value as something that is not only different than real world pursuits, but in a sense, replaces them and improves upon them.

The responses to Question 4 indicate that time is an important factor in establishing the value of a gaming experience. One half expressed time value in terms of the level of difficulty or ease in basic and complex gaming duties/sequences/tasks. These respondents consistently complained about "boring tasks" or if certain tasks "took too long". This indicates that time value is tied to both gamer skill and to game design. The second half of respondents commented on a very different phenomena, what I call perceived time, where time value was high if the time spent playing seemed to pass more quickly than in the real. Respondents expressed this situation in terms that usually degraded the slow passage of time in the real world, as well as how certain real world pursuits (particularly work) seemed to pass more slowly than other real world pursuits (like "standing in line at the bank". Many likened gaming to vacation or travel time, where time seemed to pleasurably stand still. The relational quality of time value in gaming then seems to both be based on time within the game itself and time in the real world. This may account for the popularity of certain games (Everquest and World of Warcraft were mentioned specifically 34 times) in the respondents' eyes; each of these games provided a high level of 'disappearance' of time and featured very little repetition and quotidian tasking.

In a general sense, online gaming was accorded a high level of value in Questions 2 - 4, largely based on the split in conceptualization of value found in Question 1. In other words, understanding value in economic terms was expressed in terms of the quantitative value of gaming and understanding value in social terms was expressed in terms of the qualitative value of gaming.

\section{Conclusion}

In The Sublime Object of Ideology, Zizek (1989) writes, "The notion of social fantasy is therefore a necessary counterpart to the concept of antagonism: fantasy is precisely the way the antagonistic fissure is masked. In other words, fantasy is a means for an ideology to take its own failure into account in advance (author's emphasis)" (p. 126). From the responses of this survey, it seems clear that online gaming, in a similar fashion, functions as a fantasy-machine so that the players need not formally establish value in gaming, but instead they manufacture value in gaming in relation to the real. Through the gaming experience, the player enters into a transaction where other pursuits become naturally tinged with less value, or, potentially, more failure. This situation is complicated by the fact that games operate on ideological levels, particularly in the face of massively-populated social arenas that often generate rules and regulations in opposition to surrounding cultural and ideological norms.

Through the responses collected in this study it appears that gaming value does not operate hermetically; it is established in relation to real world value systems. This leads to another central point-social matrices in online gaming consistently invade and are invaded by the real, particularly when gamers express the quality of gaming in relation to the quality of real world pursuits. Finally, it seems clear that online gaming, in a long line of other media forms, serves as a powerful force for the production of fantasy, and, conversely, as an equally powerful system of desire and longing for the different, the other, and the inescapable pursuit (and unavoidable failure) to achieve the most slippery of negotiations in postmodern capitalism - the total integration of the internal and external, the self and surrounding culture.

\section{References}

Ball-Rokeach, S., \& DeFleur, M. (1976). A Dependency Model of Mass-Media Effects. Communications Research, 3, 3-21. http://dx.doi.org/10.1177/009365027600300101

Black, A. (2001). Modern American Queer History. Philadelphia: Temple University Press. 
Caillois, R. (1961). Man, Play and Games (M. Barash, Trans.). New York: Free Press of Glencoe.

Castronova, E. (2006). On the Research Value of Large Games: Natural Experiments in Norrath and Camelot. Games and Culture, 1, 163-186. http://dx.doi.org/10.1177/1555412006286686

Creedy, J. (2007). Development of the Theory of Exchange. Cheltenham, UK: Edward Elgar.

Huizinga, J. (1945). Homo Ludens. New York: Harper and Row.

Katz, M., \& Foulkes, D. (1962). On the Use of Mass Media as “Escape:” Clarification of a Concept. The Public Opinion Quarterly, 26, 377-388. http://dx.doi.org/10.1086/267111

Malaby, T. (2006). Parlaying Value: Capital in and Beyond Virtual Worlds. Games and Culture, 1, 141-162. http://dx.doi.org/10.1177/1555412006286688

Marx, K. (1968). The Communist Manifesto (P. M. Sweezy, Trans.). New York: Monthly Review Press.

Messner, M. (1992). Power at Play. Boston, Beacon.

Odum, H. (1983). Systems Ecology: An Introduction. New York: John Wiley and Sons.

Phelan, P., \& Lane, J. (1998). The Ends of Performance. New York: NYU Press.

Smith, A. (1937). The Wealth of Nations. New York: The Modern Library.

Zizek, S. (1989). The Sublime Object of Ideology. London: Verso. 
Scientific Research Publishing (SCIRP) is one of the largest Open Access journal publishers. It is currently publishing more than 200 open access, online, peer-reviewed journals covering a wide range of academic disciplines. SCIRP serves the worldwide academic communities and contributes to the progress and application of science with its publication.

Other selected journals from SCIRP are listed as below. Submit your manuscript to us via either submit@scirp.org or Online Submission Portal.
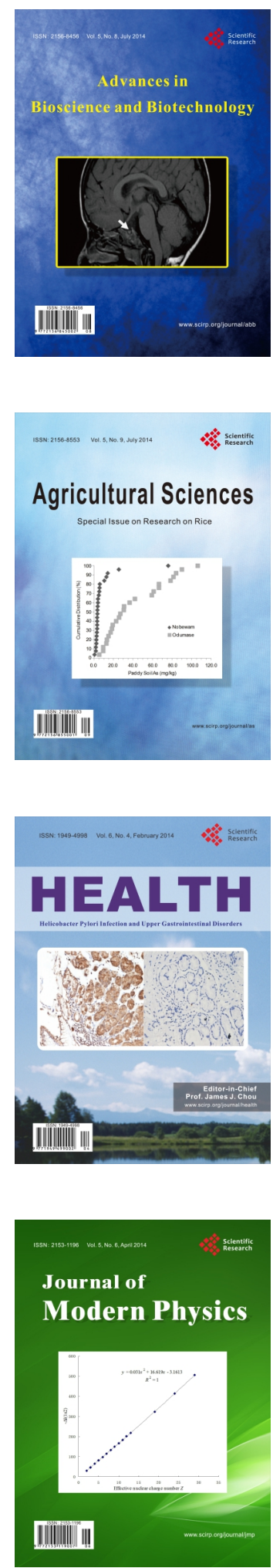
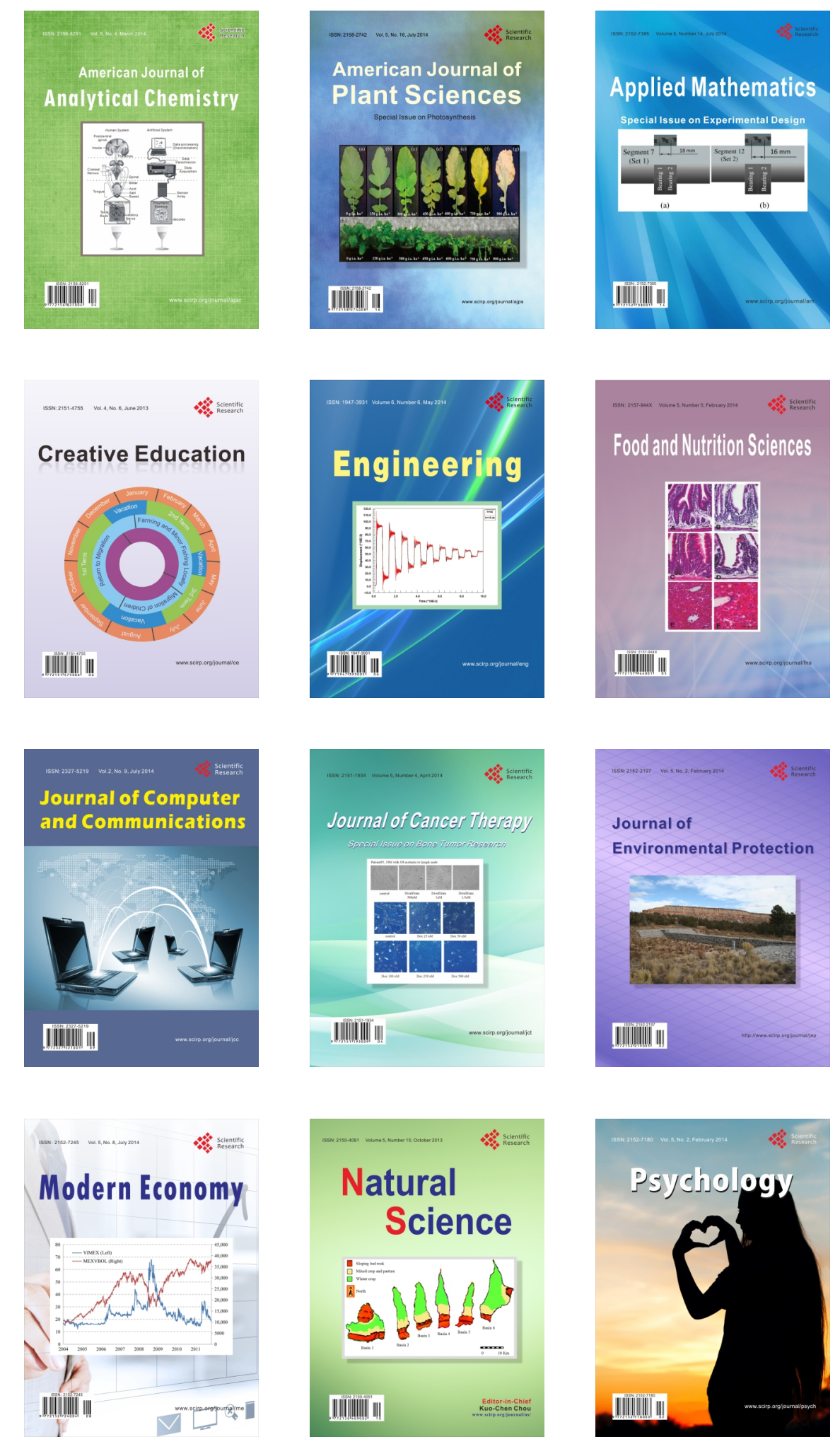